\title{
Patients' self-reported adherence to capecitabine on XELOX treatment in metastatic colorectal cancer: findings from a retrospective cohort analysis
}

\author{
This article was published in the following Dove Press journal: \\ Patient Preference and Adherence \\ 9 April 2015 \\ Number of times this article has been viewed
}

\author{
Kazuyoshi Kawakami' \\ Eri Nakamoto' \\ Takashi Yokokawa' \\ Kazuo Sugita' \\ Yutarou Mae' \\ Akane Hagino' \\ Mitsukuni Suenaga ${ }^{2}$ \\ Nobuyuki Mizunuma ${ }^{2}$ \\ Sayaka Oniyama ${ }^{3}$ \\ Yoshiaki Machida ${ }^{3}$ \\ Toshiharu Yamaguchi ${ }^{2}$ \\ Toshihiro Hama' \\ 'Department of Pharmacy, \\ ${ }^{2}$ Gastroenterology Center, Cancer \\ Institute Hospital, Japanese \\ Foundation for Cancer Research, \\ ${ }^{3}$ Section for Practical Education, \\ Hoshi University School of Pharmacy \\ and Pharmaceutical Sciences, \\ Tokyo, Japan
}

Background: Capecitabine plus oxaliplatin (XELOX) has been established as a first-line treatment for metastatic colorectal cancer. Adherence is particularly important with capecitabine to maintain appropriate curative effect. In this study, we monitored the adherence to capecitabine on XELOX treatment and investigated which factors might decrease compliance.

Methods: The study included 242 consecutive patients who received XELOX treatment for metastatic colorectal cancer between October 2009 and March 2012. Adherence to capecitabine was checked by pharmacists with a patient-reported treatment diary at a pharmaceutical outpatient clinic. Adherence rate was defined as the number of times that a patient took capecitabine in a 14-day cycle/28 prescribed doses. We retrospectively surveyed median relative dose intensities of capecitabine and the factors deteriorating adherence across eight cycles from electronic patient records and examined differences in compliance rates according to age.

Results: The study included 144 male and 98 female patients. The overadherence rate was $1.5 \%$ $(n=23)$. The median adherence rate was $93.5 \%(n=242)$ in the first cycle of XELOX treatment, which gradually rose to $96.1 \%(n=148)$ in the eighth cycle. The median relative dose intensity of capecitabine was $79.2 \%$. The main factors contributing to decreased adherence to capecitabine were diarrhea (22.5\%, 352 instances) and nausea/vomiting (13.8\%, 215 instances). The rate of missed dose was $12.1 \%$. Analysis of adherence issues in relation to patient age showed a trend toward worse adherence to capecitabine therapy in the group of patients aged $\geq 80$ years (hazard ratio $=3.83 ; 95 \%$ confidence interval $2.48-5.91, P<0.001$ versus $70-80$ years group and versus $<70$ years group, chi-square test).

Conclusion: Patient-reported adherence to capecitabine on XELOX treatment in clinical practice is high but adversely affected by side effects. Patients aged 80 years or more exhibit a significant decrease in compliance compared with younger patients.

Keywords: pharmaceutical outpatient clinic, oral anticancer drugs

\section{Introduction}

The use of oral anticancer agents has increased in modern oncology treatment: $25 \%$ of cancer chemotherapy currently in development can be taken orally. ${ }^{1}$ The use of oral anticancer drugs improves the quality of life of cancer patients by reducing hospital stay and giving them a greater sense of control over their treatment, ${ }^{2}$ but it poses important challenges such as managing side effects, prolonged treatment period, and adherence issues.

Patient adherence is defined as the number of doses taken as a percentage of the number expected. Adherence to prescribed treatment regimens for chronic
Correspondence: Kazuyoshi Kawakami Japanese Foundation for Cancer Research, Cancer Institute Hospital, Department of Pharmacy, 3-8-3I Ariake Koto-ku, Tokyo 135-8550, Japan Email kazu.kawakami@jfcr.or.jp 
nononcologic diseases averages $<80 \%$. $^{3,4}$ Patients with cancer, because it is a life-threatening disease, are expected to be particularly adherent to medication. ${ }^{5-7}$ Several studies have been published investigating patient adherence to capecitabine; median adherence across all cycles and studies was 78\%. ${ }^{8,9}$ Winterhalder et $\mathrm{al}^{5}$ used participant self-reports to explore adherence in patients with gastrointestinal and breast cancer.

Patients in the present study were treated with the chemotherapeutic agent capecitabine (Xeloda; Hoffmann-La Roche Ltd., Basel, Switzerland), a fluoropyrimidine that is an orally administered prodrug of cytotoxic fluorouracil (5-FU). Capecitabine, which is frequently used in the treatment of colorectal or gastric cancer, has improved tolerability and comparable efficacy compared with infusional or bolus $5-\mathrm{FU}^{10}$ and has similar efficacy to bolus 5-FU/folinic acid in the first-line treatment of metastatic colorectal cancer. ${ }^{1-13}$ XELOX, a regimen combining capecitabine and oxaliplatin, consists of the standard 21-day intermittent schedule (ie, 14 days on followed by 7 days off) of capecitabine combined with oxaliplatin on day 1 .

Adherence is crucial for the success of oral anticancer agent therapy. However, many oncologists, pharmacists, and nurses do not check for oral anticancer agent adherence in ambulatory practice, even though understanding the reasons for nonadherence is essential for the development of interventions to improve adherence. In this study, adherence to capecitabine during XELOX treatment was checked by pharmacists via a patient-reported treatment diary at our pharmaceutical outpatient clinic for metastatic colorectal cancer. The aim of the study was to evaluate real-life adherence via retrospective cohort analysis of Japanese patients undergoing XELOX treatment.

\section{Materials and methods Study design}

Enrollment criteria comprised patients who received XELOX treatment for metastatic colorectal cancer between October 1, 2009 and March 31, 2012 at the Cancer Institute Hospital, Japanese Foundation for Cancer Research and who attended a pharmaceutical outpatient clinic. We included 242 consecutive patients. Adherence to capecitabine was checked by pharmacists with a patient-reported treatment diary at the pharmaceutical outpatient clinic. Adherence rate was defined as the number of times that a patient took capecitabine in a 14-day cycle/28 doses prescribed. The Clinical Research Ethics Review Committee of the Japanese Foundation for
Cancer Research, Cancer Institute Hospital approved this research (approval no: 2012-1035).

\section{Data collection}

Adherence to capecitabine was checked by pharmacists via a patient-reported treatment diary. The reasons for nonadherence were established by these pharmacists. We retrospectively surveyed median relative dose intensities $^{14}$ of capecitabine and the factors deteriorating compliance over eight treatment cycles using electronic patient records. Differences in compliance rates were examined according to patient age and tested using the chi-square test.

The Bonferroni correction was used to conservatively account for multiple comparisons. All $P$-values reported were two-sided, and a level of 0.05 was considered statistically significant. Statistical analyses were performed using SPSS, version 11.5.

\section{Results}

\section{Patient characteristics}

The study included 144 male and 98 female patients (Table 1). Their mean age was 63 years (range 27-86). All patients were diagnosed with metastatic colorectal cancer and most (92.9\%) had an Eastern Cooperative Oncology Group (ECOG) performance status of $0 ; 17$ patients $(7.1 \%)$ had an ECOG performance status of 1. Capecitabine was prescribed as XELOX + bevacizumab treatment for 162 (66.9\%) participants while $80(33.1 \%)$ received XELOX

Table I Baseline patient characteristics

\begin{tabular}{lll}
\hline Characteristics & Patients $(\mathbf{n}=\mathbf{2 4 2})$ & $\%$ \\
\hline Sex & 144 & 59.5 \\
$\quad$ Male & 98 & 40.5 \\
Female & & \\
Age, years & 63 & \\
$\quad$ Median & $27-86$ & \\
Range & & 92.9 \\
ECOG performance status & 225 & 7.1 \\
0 & 17 & 0 \\
I & 0 & \\
2 & & 52.9 \\
Primary tumor site & 128 & 36.3 \\
Colonic & 88 & 6.2 \\
Rectal & 15 & 4.5 \\
Cecal & 11 & \\
Appendiceal & & 66.9 \\
Chemotherapy & 162 & 33.1 \\
$\quad$ XELOX + bevacizumab & 80 & \\
XELOX &
\end{tabular}

Abbreviations: ECOG, Eastern Cooperative Oncology Group; XELOX, capecitabine plus oxaliplatin. 
treatment. The median relative dose intensity of capecitabine was $79.2 \%$.

\section{Self-reported adherence}

Overall, 124 patients $(51.2 \%)$ did not record any intake mistakes with capecitabine therapy and were considered fully compliant while 118 patients (48.8\%, 1,561 instances) reported some kind of compliance error. The rate of overadherence was $1.5 \%(n=23)$. Median adherence rates were $93.5 \%(n=242)$ in the first cycle of XELOX treatment and $95.5 \%(n=229)$ in the second cycle, rising to $96.1 \%(n=148)$ in the eighth cycle (Figure 1). The reasons for nonadherence were diarrhea $(22.5 \%, 352$ instances), nausea/vomiting (13.8\%, 215 instances), fever (12.7\%, 198 instances), missed dose (12.1\%, 189 instances), ileus/constipation (6.5\%, 102 instances), and progressive disease (5.6\%, 87 instances) (Figure 2). The medications most commonly used were skin moisturizer (100\%) and antidiarrheal (one-time-only dose) (100\%).

The rate of missed dose was $12.1 \%$. We categorized the patients who missed a dose of capecitabine according to the number of times it occurred: $\geq 10$ times, three patients $(1.2 \%) ; 6 \leq 9$ times, seven patients $(2.9 \%) ; 3 \leq 5$ times, nine patients $(3.7 \%)$; once or twice, 31 patients $(12.8 \%)$ in eight cycles of XELOX treatment (Figure 3 ).

\section{Relationship of self-reported adherence and age}

The reported rate of adherence to capecitabine in the first treatment cycle was $80.4 \%$ in patients aged $\geq 80$ years, $94.0 \%$ in the $70-80$ years group, and $93.8 \%$ for patients aged $<70$ years (Figure 4 ). Median relative dose intensities of capecitabine were $57.3 \%$ in the group of patients aged $\geq 80$ years, $77.0 \%$ in the $70-80$ years group, and $82.0 \%$ in patients aged $<70$ years. An analysis of the probability of adherence issues in relation to patient age revealed a trend toward better adherence with capecitabine therapy in younger patients (hazard ratio $=3.83 ; 95 \%$ confidence interval $2.48-5.91, P<0.001$ versus $70-80$ years group and versus $<70$ years group; chi-square test).

\section{Discussion}

This research identified the adherence rate to capecitabine, and the factors that decrease it, at a pharmaceutical outpatient clinic over eight treatment cycles. Especially, overadherence was $1.5 \%$ and $12.1 \%$ of doses were missed; $4.1 \%$ of patients made five or more errors. Adherence was statistically significantly lower in patients aged $\geq 80$ years compared with younger patients. ( $P<0.001$ versus $70-80$ years group and versus $<70$ years group; chi-square test).

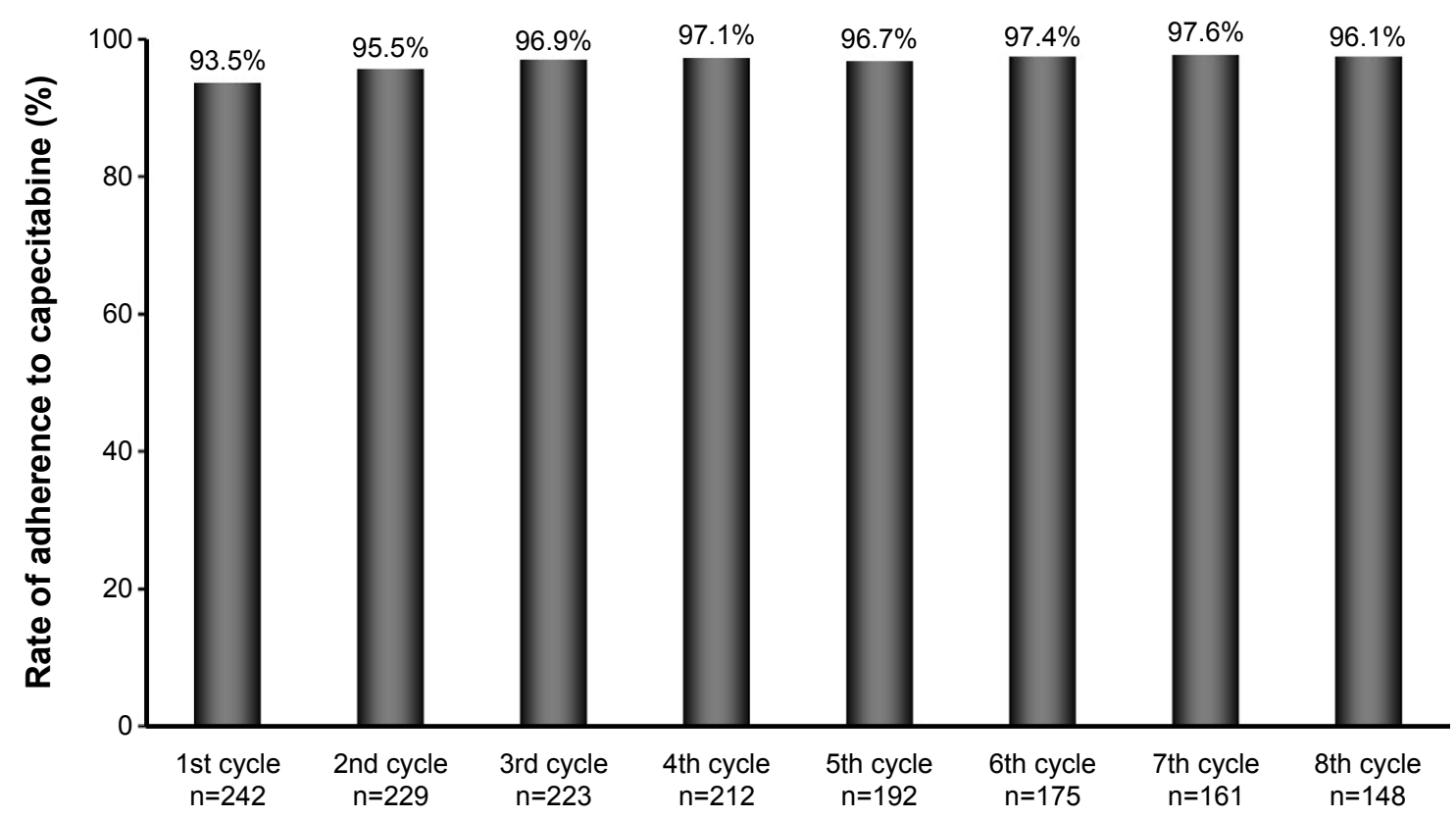

Figure I Capecitabine adherence rate on XELOX treatment.

Notes: Adherence rate with capecitabine during cycles I-8; Adherence to capecitabine was checked by pharmacists via the patients' self-reported treatment diaries at a pharmaceutical outpatient clinic; The reason for the number of patients decreasing through the cycles is deviation from XELOX therapy.

Abbreviation: XELOX, capecitabine plus oxaliplatin. 


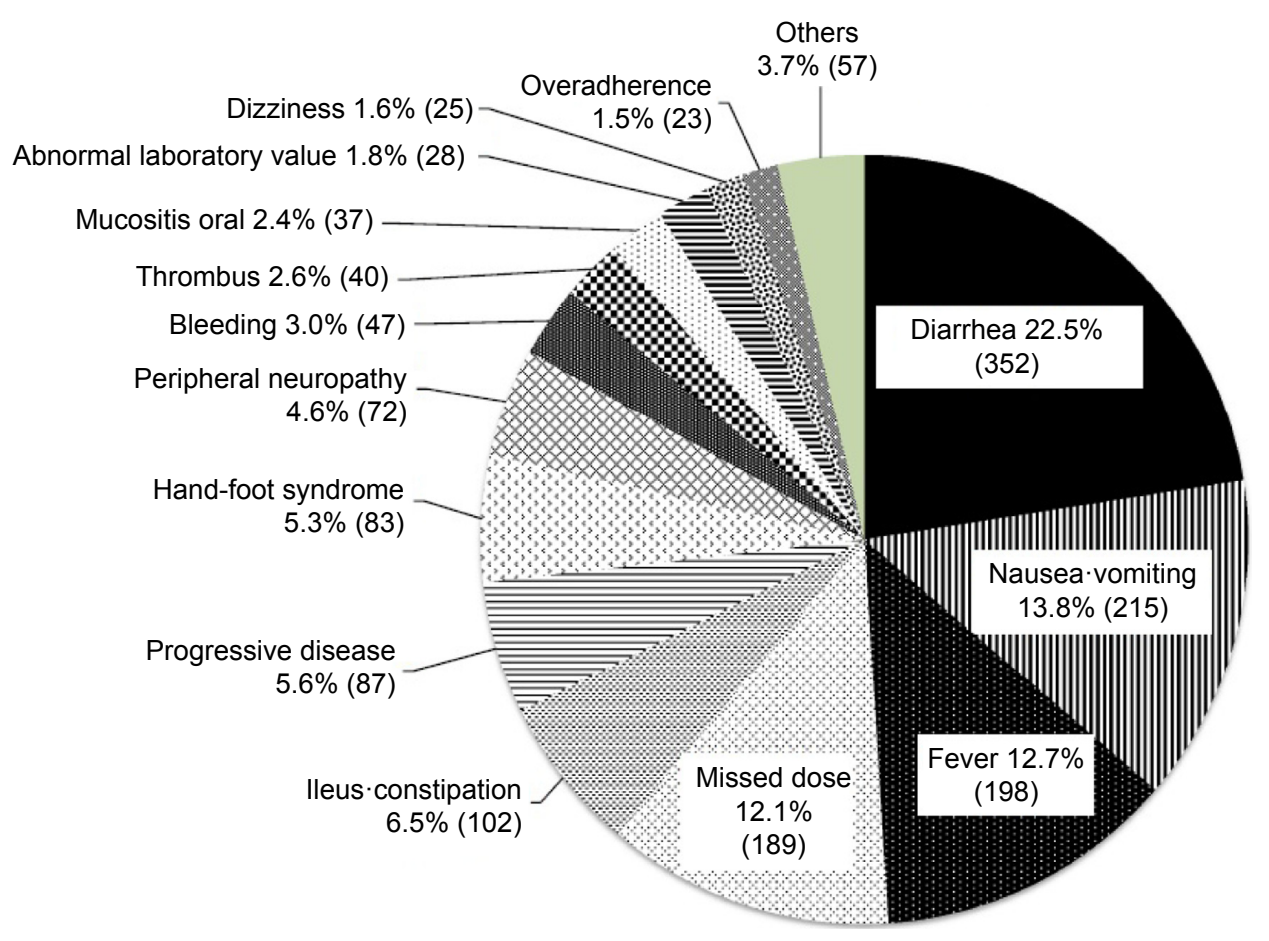

Figure 2 Factors reducing adherence to capecitabine on XELOX treatment $(n=I, 56 I)$.

Note: Factors reducing adherence to capecitabine during cycles I-8.

Abbreviation: XELOX, capecitabine plus oxaliplatin.

Cancer patients are generally expected to have higher adherence to treatment than other patients because they are highly motivated by the gravity of their disease. ${ }^{15,16}$ However, studies have shown cancer patients to have similar adherence rates to patients with other diseases. ${ }^{17-19}$ Treatment duration plays a role in adherence to the regimen: when medication is continued over a longer period of time, patients become less adherent. ${ }^{20}$ Most of the studies included heterogeneous target tumor types, regimens, and therapy settings. ${ }^{21}$

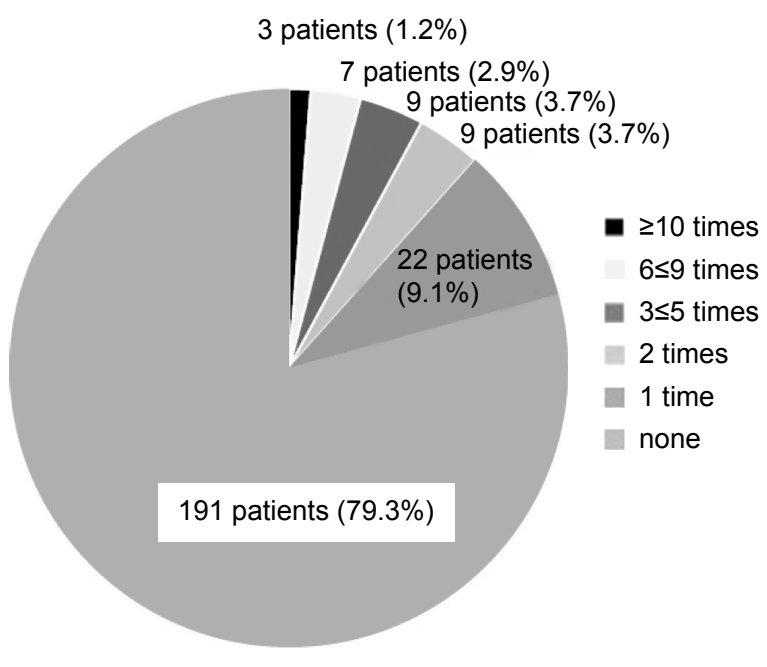

Figure 3 Missed-dose rates with capecitabine.
Our study focused on metastatic colorectal cancer and on XELOX treatment in clinical practice. We have explored the problem of medication adherence in cancer patients; our data in a limited tumor type and regimen will be highly useful and valuable.

One of the major limitations to medication event monitoring systems is that they are feasible in only some health care systems. Also, they are not suitable for oral cytotoxic agents, which require close monitoring of side effects and regular patient visits. There is no gold-standard measurement, and all methods have limitations. ${ }^{17,22}$ Previous studies of oral cytotoxic chemotherapeutic drugs have mainly used self-reported questionnaires, ${ }^{23}$ which tend to overestimate adherence because patients are inclined to over-report to please their doctors. It seems likely that patient-completed diaries may be less influenced by this issue, because the patient is asked to record each dose as it is taken. Therefore, we used self-reported adherence based on many patient-completed medication diaries.

Associated factors influencing nonadherence to oral anticancer drugs are multifactorial and interrelated. ${ }^{24,25}$ Treatment-related side effects are the most frequently reported therapy-related factors associated with nonadherence to capecitabine, ${ }^{5}$ and age is another predominant factor. ${ }^{21}$ Our study indicated that nonadherence was correlated with increasing number of adverse effects. The most common 


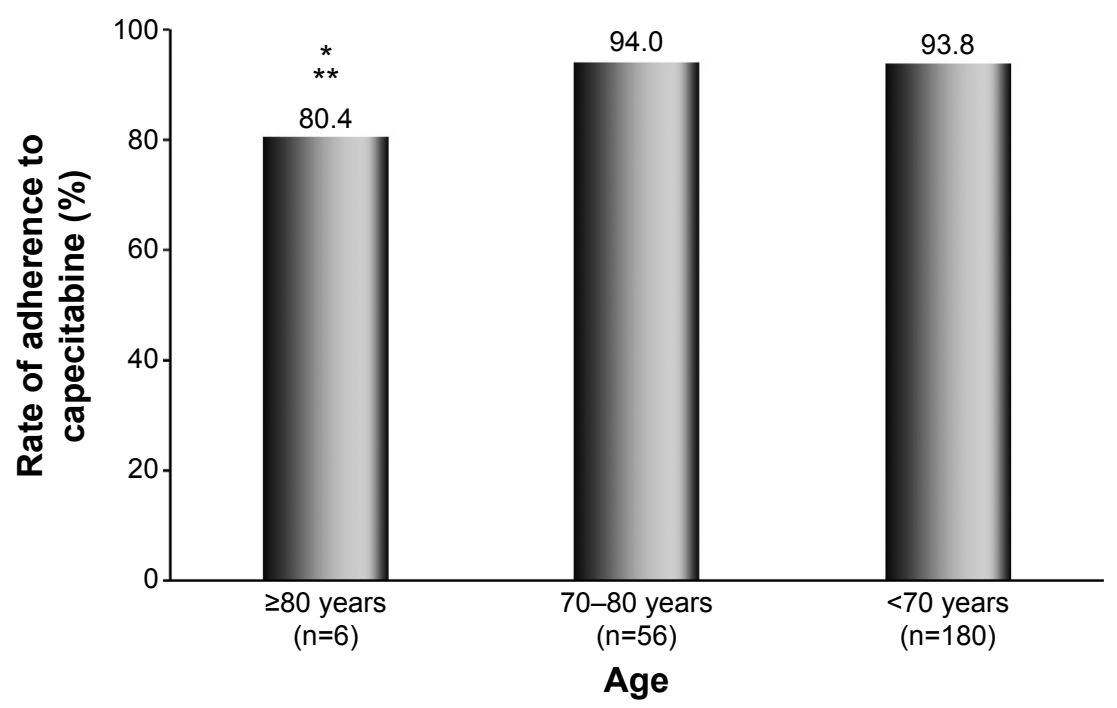

Figure 4 Effect of age on adherence to capecitabine.

Notes: Adherence to capecitabine in the first cycle, by patient age. In the $\geq 80$ years group ( $n=6$ ), there were 135 doses of capecitabine taken (I68 recommended); I,474 doses were taken $(I, 568$ recommended) in the $70-80$ years group $(n=56) ; 4,725$ doses were taken $(5,040$ recommended) in the $<70$ years group $(n=180)$; $* P<0.00$ I compared with the $70-80$ years group; $* * P<0.001$ compared with the $<70$ years group.

side effects of capecitabine are gastrointestinal disorders (diarrhea, nausea, vomiting) and hand-foot syndrome. We prescribed supportive therapy for all cases in this study beforehand, but it is thought that patients do not always use supportive treatments appropriately. Thus, it is necessary to provide patient-tailored educational support ${ }^{26,27}$ and management of symptoms during follow-up. ${ }^{28}$

The rate of missed dose was $12.1 \%$, and approximately $5 \%$ of patients habitually missed medicine. A main factor causing patients to miss a dose is eating out. However, in our study, it became apparent that more than $95 \%$ of patients had good adherence to capecitabine. Many studies provide limited information regarding the relationship between nonadherence and severity of side effects. Our data identified how often the capecitabine missed dose occurred in association with XELOX treatment in first-line treatment for metastatic colorectal cancer.

The adherence of the approximately $5 \%$ of patients who are intentionally nonadherent could be enhanced by means of advanced educational pharmaceutical care interventions. Behavioral interventions such as medication dosette boxes or alarm clocks and education about the characteristics of the drug capecitabine, possible adverse events, and their appropriate management, as well as the individual treatment regimen in detail, could be used more extensively to improve adherence in unintentionally nonadherent patients.

Self-reported compliance with capecitabine in patients with gastrointestinal and breast cancer was not influenced by age. ${ }^{5}$ However, in this study, patients aged $\geq 80$ years exhibited significantly decreased adherence compared with younger patients. Additionally, median relative dose intensities of capecitabine were not shown in previous studies; ${ }^{5}$ we found median relative dose intensities of capecitabine of $57.3 \%$ in the $\geq 80$ years group, $77.0 \%$ in the $70-80$ years group, and $82.0 \%$ in the $<70$ years group. We consider the findings from this study to be an important part of understanding lower relative dose intensity and adherence with capecitabine in patients aged $\geq 80$ years.

Younger ${ }^{20,29,30}$ and older age $\mathrm{e}^{19,20,31}$ have both been reported to be associated with nonadherence in patients taking oral anticancer drugs. Younger age was defined as $\leq 45$ years, ${ }^{20,29}$ older as $\geq 85$ years. ${ }^{20}$ Older age was also found to be associated with lower persistence with the oral anticancer drug treatment. In this study, patients aged $\geq 80$ years exhibited significantly lower compliance compared with younger patients. Patient eligibility in a previous randomized Phase III study of XELOX as first-line therapy for metastatic colorectal cancer was $\geq 18$ years, with the oldest enrolled patient aged 86 years. ${ }^{30}$ Those aged $\geq 80$ years had lower compliance with capecitabine than younger participants; nevertheless, the median relative dose intensity of capecitabine in the $\geq 80$ years group was $57.3 \%$ in our study. In the case of patients aged $\geq 80$ years, a switch to injection therapy, FOLFOX, might be considered in some cases. It became clear in our study that adherence in younger patients was high (90\%). A major limitation of our study is the relatively small number of patients aged $>80$ years. Further research should include more elderly patients. 
Checking adherence to oral anticancer drugs via pharmaceutical outpatient clinics is of considerable clinical importance. Pharmacist interventions can significantly improve medication adherence. ${ }^{32}$ An oral chemotherapy management clinic service by a pharmacist was effective in delivering early interventions, resulting in decreased rates of adverse effects, nonadherence, drug interactions, and medication errors over time. ${ }^{33}$ The identification of potential predictors of adherence would facilitate the utilization and broad application of the proposed adherence screening and modular medication management. The results of this study, obtained from a pharmaceutical outpatient clinic, demonstrate the adherence rate and the factors deteriorating the compliance with capecitabine over eight treatment cycles. Patients aged 80 years and over were suggested to be statistically significantly at risk of nonadherence to capecitabine with XELOX treatment. Our data provide important information about optimizing patients' care.

\section{Disclosure}

The authors report no conflicts of interest in this work.

\section{References}

1. Banna GL, Collovà E, Gebbia V, et al. Anticancer oral therapy: emerging related issues. Cancer Treat Rev. 2010;36(8):595-605.

2. Regnier Denois V, Poirson J, Nourissat A, Jacquin JP, Guastalla JP, Chauvin F. Adherence with oral chemotherapy: results from a qualitative study of the behaviour and representations of patients and oncologists. Eur J Cancer Care (Engl). 2011;20(4):520-527.

3. Sabate E, editor. Adherence to long-term therapies: evidence for action [webpage on the Internet]. Geneva: World Health Organization; 2003. Available from: http://www.who.int/chp/knowledge/publications/ adherence_report/en/. Accessed January 1, 2015.

4. Cutler DM, Everett W. Thinking outside the pillbox - medication adherence as a priority for health care reform. $N$ Engl J Med. 2010;362(17):1553-1555.

5. Winterhalder R, Hoesli P, Delmore G, et al; SAEDA Investigators Group (Swiss prospective cohort group). Self-reported compliance with capecitabine: findings from a prospective cohort analysis. Oncology. 2011;80(1-2):29-33.

6. Osterberg L, Blaschke T. Adherence to medication. NEngl J Med. 2005; 353(5):487-497.

7. Haynes RB, McDonald HP, Garg AX. Helping patients follow prescribed treatment: clinical applications. JAMA. 2002;288(22): 2880-2883.

8. Partridge AH, Archer L, Kornblith AB, et al. Adherence and persistence with oral adjuvant chemotherapy in older women with early-stage breast cancer in CALGB 49907: adherence companion study 60104. J Clin Oncol. 2010;28(14):2418-2422.

9. Muss HB, Berry DA, Cirrincione CT, et al; CALGB Investigators. Adjuvant chemotherapy in older women with early-stage breast cancer. N Engl J Med. 2009;360(20):2055-2065.

10. Cassidy J, Twelves C, Van Cutsem E, et al; Capecitabine Colorectal Cancer Study Group. First-line oral capecitabine therapy in metastatic colorectal cancer: a favorable safety profile compared with intravenous 5-fluorouracil/leucovorin. Ann Oncol. 2002;13(4):566-575.
11. Van Cutsem E, Twelves C, Cassidy J, et al; Xeloda Colorectal Cancer Study Group. Oral capecitabine compared with intravenous fluorouracil plus leucovorin in patients with metastatic colorectal cancer: results of a large phase III study. J Clin Oncol. 2001;19(21):4097-4106.

12. Van Cutsem E, Hoff PM, Harper P, et al. Oral capecitabine vs intravenous 5-fluorouracil and leucovorin: integrated efficacy data and novel analyses from two large, randomised, phase III trials. Br J Cancer. 2004;90(6):1190-1197.

13. Hoff PM, Ansari R, Batist G, et al. Comparison of oral capecitabine versus intravenous fluorouracil plus leucovorin as first-line treatment in 605 patients with metastatic colorectal cancer: results of a randomized phase III study. J Clin Oncol. 2001;19(8):2282-2292.

14. Hryniuk WM, Goodyear M. The calculation of received dose intensity. J Clin Oncol. 1990;8(12):1935-1937.

15. Klein CE, Kastrissios H, Miller AA, et al. Pharmacokinetics, pharmacodynamics and adherence to oral topotecan in myelodysplastic syndromes: a Cancer and Leukemia Group B study. Cancer Chemother Pharmacol. 2006;57(2):199-206.

16. Waterhouse DM, Calzone KA, Mele C, Brenner DE. Adherence to oral tamoxifen: a comparison of patient self-report, pill counts, and microelectronic monitoring. J Clin Oncol. 1993;11(6):1189-1197.

17. Ruddy K, Mayer E, Partridge A. Patient adherence and persistence with oral anticancer treatment. CA Cancer J Clin. 2009;59(1):56-66.

18. Nilsson JL, Andersson K, Bergkvist A, Björkman I, Brismar A, Moen J. Refill adherence to repeat prescriptions of cancer drugs to ambulatory patients. Eur J Cancer Care (Engl). 2006;15(3):235-237.

19. Noens L, van Lierde MA, De Bock R, et al. Prevalence, determinants, and outcomes of nonadherence to imatinib therapy in patients with chronic myeloid leukemia: the ADAGIO study. Blood. 2009;113(22):5401-5411.

20. Partridge AH, Wang PS, Winer EP, Avorn J. Nonadherence to adjuvant tamoxifen therapy in women with primary breast cancer. J Clin Oncol. 2003;21(4):602-606.

21. Verbrugghe M, Verhaeghe S, Lauwaert K, Beeckman D, Van Hecke A. Determinants and associated factors influencing medication adherence and persistence to oral anticancer drugs: a systematic review. Cancer Treat Rev. 2013;39(6):610-621.

22. Partridge AH, Avorn J, Wang PS, Winer EP. Adherence to therapy with oral antineoplastic agents. J Natl Cancer Inst. 2002;94(9):652-661.

23. Font R, Espinas JA, Gil-Gil M, et al. Prescription refill, patient selfreport and physician report in assessing adherence to oral endocrine therapy in early breast cancer patients: a retrospective cohort study in Catalonia, Spain. Br J Cancer. 2012;107(8):1249-1256.

24. Barron TI, Connolly R, Bennett K, Feely J, Kennedy MJ. Early discontinuation of tamoxifen: a lesson for oncologists. Cancer. 2007;109(5):832-839.

25. Kahn KL, Schneider EC, Malin JL, Adams JL, Epstein AM. Patient centered experiences in breast cancer: predicting long-term adherence to tamoxifen use. Med Care. 2007;45(5):431-439.

26. Moore S. Facilitating oral chemotherapy treatment and compliance through patient/family-focused education. Cancer Nurs. 2007; 30(2):112-122; quiz 123.

27. Vermeire E, Hearnshaw H, Van Royen P, Denekens J. Patient adherence to treatment: three decades of research. A comprehensive review. J Clin Pharm Ther. 2001;26(5):331-342.

28. Kav S, Johnson J, Rittenberg C, et al. Role of the nurse in patient education and follow-up of people receiving oral chemotherapy treatment: an international survey. Support Care Cancer. 2008;16(9): 1075-1083.

29. Sedjo RL, Devine S. Predictors of non-adherence to aromatase inhibitors among commercially insured women with breast cancer. Breast Cancer Res Treat. 2011;125(1):191-200.

30. Atkins L, Fallowfield L. Intentional and non-intentional non-adherence to medication amongst breast cancer patients. Eur J Cancer. 2006; 42(14):2271-2276. 
31. Neugut AI, Subar M, Wilde ET, et al. Association between prescription co-payment amount and compliance with adjuvant hormonal therapy in women with early-stage breast cancer. J Clin Oncol. 2011; 29(18):2534-2542.

32. Morgado MP, Morgado SR, Mendes LC, Pereira LJ, Castelo-Branco M. Pharmacist interventions to enhance blood pressure control and adherence to antihypertensive therapy: Review and meta-analysis. Am J Health Syst Pharm. 2011;68(3):241-253.
33. Wong SF, Bounthavong M, Nguyen C, Bechtoldt K, Hernandez E Implementation and preliminary outcomes of a comprehensive oral chemotherapy management clinic. Am J Health Syst Pharm. 2014;71(11):960-965.

\section{Publish your work in this journal}

Patient Preference and Adherence is an international, peer-reviewed, open access journal that focuses on the growing importance of patient preference and adherence throughout the therapeutic continuum. Patient satisfaction, acceptability, quality of life, compliance, persistence and their role in developing new therapeutic modalities and compounds to optimize clinical outcomes for existing disease states are major areas of interest for the journal. This journal has been accepted for indexing on PubMed Central. The manuscript management system is completely online and includes a very quick and fair peer-review system, which is all easy to use. Visit http://www. dovepress.com/testimonials.php to read real quotes from published authors.

\footnotetext{
Submit your manuscript here: http://www.dovepress.com/patient-preference-and-adherence-journal
} 